\title{
The role of extreme orbits in the global organization of periodic regions in parameter space for one dimensional maps
}

\author{
Diogo Ricardo da Costa ${ }^{\mathrm{a}, *}$, Matheus Hansen ${ }^{\mathrm{a}, \mathrm{b}}$, Gustavo Guarise ${ }^{\mathrm{a}}$, Rene O. Medrano-T ${ }^{\mathrm{c}, \mathrm{d}}$ \\ Edson D. Leonel ${ }^{\mathrm{a}, \mathrm{e}}$ \\ a Departamento de Física, UNESP - Universidade Estadual Paulista, Av. 24A, 1515, Bela Vista, 13506-900, Rio Claro, SP, Brazil \\ ${ }^{\mathrm{b}}$ Instituto de Física, Univ. São Paulo, Rua do Matão, Cidade Universitária, 05314-970, São Paulo - SP, Brazil \\ c Departamento de Ciências Exatas e da Terra, UNIFESP - Universidade Federal de São Paulo, Rua São Nicolau, 210, Centro, 09913-030, Diadema, SP, Brazil \\ d Department of Mathematics, Imperial College London, London SW7 2AZ, UK \\ e Abdus Salam International Center for Theoretical Physics, Strada Costiera 11, 34151 Trieste, Italy
}

\section{A R T I C L E I N F O}

\section{Article history:}

Received 6 October 2015

Received in revised form 15 January 2016

Accepted 27 February 2016

Available online 2 March 2016

Communicated by C.R. Doering

\section{Keywords:}

Extreme orbits

One dimensional maps

Parameter space

\begin{abstract}
A B S T R A C T
We show that extreme orbits, trajectories that connect local maximum and minimum values of one dimensional maps, play a major role in the parameter space of dissipative systems dictating the organization for the windows of periodicity, hence producing sets of shrimp-like structures. Here we solve three fundamental problems regarding the distribution of these sets and give: (i) their precise localization in the parameter space, even for sets of very high periods; (ii) their local and global distributions along cascades; and (iii) the association of these cascades to complicate sets of periodicity. The extreme orbits are proved to be a powerful indicator to investigate the organization of windows of periodicity in parameter planes. As applications of the theory, we obtain some results for the circle map and perturbed logistic map. The formalism presented here can be extended to many other different nonlinear and dissipative systems.
\end{abstract}

(c) 2016 Elsevier B.V. All rights reserved.
After the seminal paper of Arnold [1] discussing the organization of the Arnold tongues, the interest and hence the understanding of windows of periodicity surrounded by chaotic regions in parameter planes has increased significantly. Thanks to the advances and contributions of Gallas [2], defining the shrimp-like terminology in parameter planes of dissipative systems, the subject becomes popular and many different important results were obtained [3-6]. More advances including also spirals structures [7,8], these characterized via Lyapunov exponents, were also discussed $[9,10]$. The interest goes beyond the theoretical investigation and a recent experimental confirmation of such structures was discussed by Stoop et al. [11].

The complexity for a continuous set of periodic structure in parameter space can be described by using bifurcation theory [12]. However, this theory fails to explain the collective scenario, excluding Shilnikov systems since the spirals observed in the parameter planes of such systems are produced via homoclinic bifurcations [13-15]. There are indeed infinite cascades of complex structures scattered over the parameter space [16-18]. In regions

\footnotetext{
* Corresponding author.

E-mail address: diogo_cost@hotmail.com (D.R. da Costa).
}

of quasiperiodicity, where the system is invertible, the organization of the Arnold tongues can be understood by the number theory [1]. However, there are few and limited explanations of the periodicity for the majority of shrimps observed inside of chaotic regions. A discussion regarding the period-adding cascades can be found in Refs. [19-21] while, Lorenz [22], in his last paper, discussed a local organization for such structures in the Hénon map. Nevertheless, the scenario of the periodic regions is completely different from one system to the other, therefore being an open problem. As a main goal of given a full explanation of this open problem, a new approach and description is necessary to explore the global organization of complexity of such periodic structures in the parameter space. Our approach discussing the role of the extreme orbits fills this lack.

To investigate the organization of periodic structures, including shrimp-like ones, in the parameter plane, we introduce extreme orbits which are limited orbits that eventually compose superstable orbits (periodic orbits with very high stability). In such plane, extreme orbits occur along curves that are able to find points where complex sets are formed due the crossing between superstable curves: Extreme curves are also crossing in these special interception points. We show that cascades of periodicity sets follow 
these extreme curves in any scale of the parameter space, bringing to light the full organization of complex sets of periodicity and shrimps.

\section{Extreme orbits in one dimensional maps}

Consider a general map

$x_{n+1}=F\left(x_{n}\right)$,

with 2 or more parameters and $x \in \mathbb{R}$. A period $k$ orbit $x_{n}=$ $F^{(k)}\left(x_{n}\right)$ is a superstable orbit if $\frac{d}{d x_{n}} F^{(k)}\left(x_{n}\right)=0$, where

$\frac{\mathrm{d}}{\mathrm{d} x_{n}} F^{(k)}\left(x_{n}\right)=\prod_{t=0}^{k-1} F^{\prime}\left(F^{(t)}\left(x_{n}\right)\right)$,

where $F^{(0)}\left(x_{n}\right) \equiv x_{n}$ [23]. Therefore, considering the first term $F^{\prime}\left(x_{n}\right)$ of the sequence of products, given by $t=0$ in Eq. (2), we conclude that superstable orbits contain, at least, one of the solutions $\left\{x_{1}^{*}, x_{2}^{*}, \ldots, x_{i}^{*}, \ldots\right\}$ of

$F^{\prime}\left(x_{n}\right)=0$.

Equation (3) may lead to classify the fixed point as an inflection point if $F^{\prime \prime}\left(x_{n}\right)=0$, but often corresponds to a local extreme points of the map, where $F^{\prime \prime}\left(x_{n}\right) \neq 0$. For this reason, we call $k$-extreme orbits, trajectories that connect solutions of Eq. (3) after $k$ iterations (even when they are inflection points), i.e., orbits that contain $x_{i}^{*}$ and $x_{j}^{*}$, such that

$x_{i}^{*}=F^{(k)}\left(x_{j}^{*}\right)$.

If an extreme point is part of a periodic orbit, then it is a superstable periodic orbit. Trivially, if $i=j$ a $k$-extreme orbit is a period $k$ superstable orbit since $x_{i}^{*}=F^{(k)}\left(x_{i}^{*}\right)$. For $i \neq j$, there is a superstable orbit when $k_{1}$ and $k_{2}$-extreme orbits, $x_{i}^{*}=F^{\left(k_{1}\right)}\left(x_{j}^{*}\right)$ and $x_{j}^{*}=F^{\left(k_{2}\right)}\left(x_{i}^{*}\right)$, respectively, occur at the same time, since $x_{i}^{*}=F^{\left(k_{1}\right)}\left(x_{j}^{*}\right)=F^{\left(k_{1}\right)}\left(F^{\left(k_{2}\right)}\left(x_{i}^{*}\right)\right)=F^{\left(k_{1}+k_{2}\right)}\left(x_{i}^{*}\right)$. In this case, the superstable orbit has period $k_{1}+k_{2}$.

In planar spaces of parameter, a general extreme orbit describes curves called extreme curves. According to the later discussion, for $x_{i}^{*}=x_{j}^{*}$ a $k$-extreme curve (set of parameters for which a $k$-extreme orbit exists) is a $k$-superstable curve. It is well known that when two superstable curves are crossing each other with a same superstable orbit, the parameter space changes drastically and a complex set of periodicity takes place in the surrounded region $[7,11,24]$. Our interest is to find how these complex sets of periodicity are organized in the parameter space. To do this, we follow $k_{1}$ and $k_{2}$-extreme curves wherein $x_{i}^{*}=F^{\left(k_{1}\right)}\left(x_{j}^{*}\right)$ and $x_{j}^{*}=F^{\left(k_{2}\right)}\left(x_{i}^{*}\right)$ with $x_{i}^{*} \neq x_{j}^{*}$, respectively. A period $k_{1}+k_{2}$ superstable orbit is formed at each crossing point of these curves. Note that this superstable orbit is associated to both $x_{i}^{*}$ and $x_{j}^{*}$ by $x_{i}^{*}=F^{\left(k_{1}+k_{2}\right)}\left(x_{i}^{*}\right)$ and $x_{j}^{*}=F^{\left(k_{1}+k_{2}\right)}\left(x_{j}^{*}\right)$, respectively. Therefore, this crossing point is also crossing two $\left(k_{1}+k_{2}\right)$-superstable curves associated to $x_{i}^{*}$ and $x_{j}^{*}$ and a complex set of periodicity is identified. As an application of the extreme curves theory we present two examples, an autonomous and a non-autonomous system, respectively, the circle map and the time perturbed logistic map.

\section{The circle map}

The circle map is given by [1]

$x_{n+1}=x_{n}+m-\frac{l}{2 \pi} \sin \left(2 \pi x_{n}\right)$,
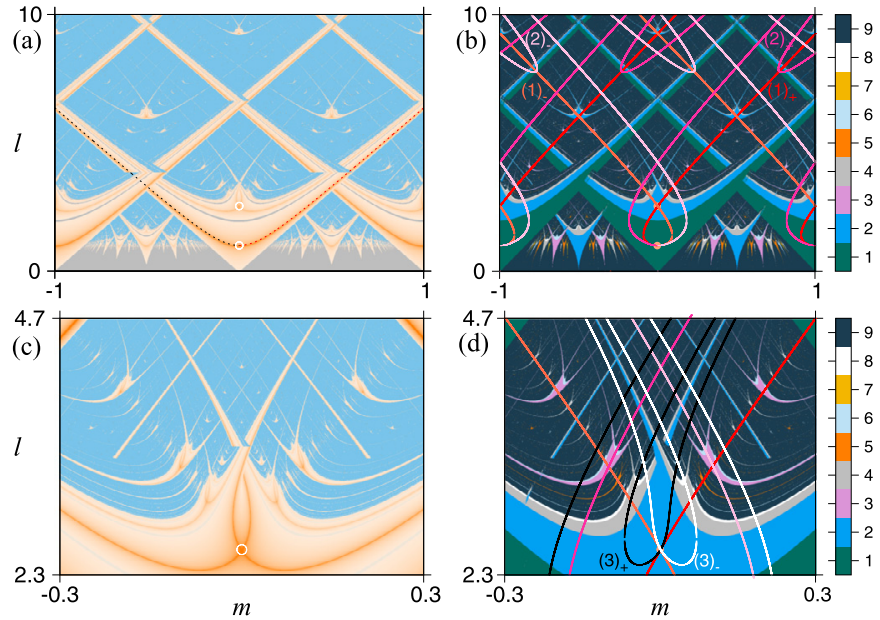

Fig. 1. (Color online.) Diagrams of $l$ vs. $m$. In (a) and (c) the pictures are colored according to the Lyapunov exponent $\lambda$. The dynamic behavior is chaotic in the blue region $(\lambda>0)$ and quasiperiodic in the gray region $(\lambda=0)$. From orange $(\lambda<0)$ to dark orange $(\lambda \ll 0)$ the stability of periodic orbits grows to superstable orbits. Superstable curves meet in the center of the white rings. The red and the black dashed lines correspond to superstable curves associated to $x_{+}^{*}$ and $x_{-}^{*}$, respectively. In (b) and (d) the colors indicate the period of the orbits. Period 9 or greater are not distinguished. The colored curves are extreme curves and the solid circles are in the same position of the white rings.

where $l$ and $m$ are control parameters of the map. Diagrams of $l \times m$, according to the maximum Lyapunov exponent $\lambda$, are shown in Figs. 1(a) and (c). The behavior is periodic in the orange region $(\lambda<0)$ where the superstability is detached by the dark orange curves $(\lambda \ll 0)$. The gray region in the bottom of picture (a) corresponds to quasiperiodic behavior $(\lambda=0)$. The periodic regions, Arnold tongues, are embedded in the quasiperiodic regime, while in the blue region chaos $(\lambda>0)$ is dominating, shrimps are observed.

Here, solutions of Eq. (3) are written as

$x_{ \pm}^{*}= \pm \frac{1}{2 \pi} \cos ^{-1}\left(\frac{1}{l}\right)+p$

where $p \in \mathbb{Z}$. Given there are infinite solutions, we focus in low values of $p$, starting the study with $p=0$. Therefore, the extreme curves are characterized by orbits wherein $x_{+}^{*}=F^{\left(k_{1}\right)}\left(x_{-}^{*}\right)$ or $x_{-}^{*}=F^{\left(k_{2}\right)}\left(x_{+}^{*}\right)$. In Figs. 1(b) and (d) there are some extreme curves, numerically determined, labeled as $(k)_{ \pm}$where $k$ indicates the number of iterations and the subscript denotes the initial condition of the orbit. Thus $(1)_{+}$indicates the extreme curve that starts at $x_{+}^{*}$ and ends at $x_{-}^{*}$ after one evolution of Eq. (5). The colors indicate the period of the orbits according to the scale in the right side of the pictures. For the circle map, periodic orbits are found for $x \bmod (1)$. Periods equal or larger than 9 are not distinguished of the chaotic region. In this diagram, one can observe that the extreme orbits $\left(k_{1}\right)_{+}$and $\left(k_{2}\right)_{-}$are crossing each other where a complex set with period $\left(k_{1}+k_{2}\right)$ is observed. Additionally, comparing Figs. 1(c) and (d), we observed that the orange curve (1) $)_{-}$crosses the red one $(1)_{+}$exactly in the same point where the period 2 superstable curves are crossing each other. The extreme curves $(1)_{ \pm}$and $(2)_{ \pm}$, which come from the Arnold tongues at $m= \pm 1$, are determined with $p= \pm 1$.

Superstable and extreme curves can be also determined analytically. The case $x_{ \pm}^{*}=F\left(x_{ \pm}^{*}\right)$ leads to

$m= \pm \frac{\sqrt{l^{2}-1}}{2 \pi}$,

where the positive (negative) solution is the superstable curve related to $x_{+(-)}^{*}$ corresponding to the red (black) dashed line in 
Fig. 1(a). According to Eq. (7), both curves join each other at $(m, l)=(0,1)$, identified by the lower white ring in Fig. 1(a). Some previous results about superstable curves in the sine map are found in [25]. On the other hand, the case where $x_{+}^{*}=F\left(x_{-}^{*}\right)$ and $x_{-}^{*}=F\left(x_{+}^{*}\right)$ results in

$m=\mp\left[\frac{1}{\pi} \cos ^{-1}\left(\frac{1}{l}\right)-\frac{\sqrt{l^{2}-1}}{2 \pi}\right]$,

where the negative (positive) solution is the extreme curve related to $x_{+(-)}^{*}$ and corresponds to the red (orange) line $(1)_{+(-)}$that departs from the central Arnold tongue in Fig. 1(b). These curves, according to Eq. (8), intercept each other in $(m, l)=(0,1)$ and $(m, l)=(0,2.5366)$, which are in the center of the white rings in Figs. 1(a) and (c) and the solid circles in Figs. 1(b) and (d). Note that, for $(m, l)=(0,1)$, we obtained the case $x_{+}^{*}=x_{-}^{*}\left[x_{ \pm}^{*}=0\right.$, for $p=0$ in Eq. (6)] and, therefore, a period $k=1$ superstable orbit is formed. We remark that this parameter set is the same point where the superstable curves [red and black dashed lines in Fig. 1(a)] meet each other. Moreover, for $(m, l)=(0,2.5366)$, it is obtained the case $x_{+}^{*} \neq x_{-}^{*}\left(x_{ \pm}^{*}= \pm 0.1855\right)$, and a period $k_{1}+k_{2}=2$ superstable orbit is formed. Figs. 1(c) and (d) show that, in this point, the superstable curves of period 2 are also crossing. Both results are in complete agreement with the previous discussion about extreme curves.

\section{The time perturbed logistic map}

The time perturbed logistic map is written as [26,27]

$x_{n+1}=x_{n} R\left(1+b_{n} \epsilon\right)\left(1-x_{n}\right)$,

where $b_{n}$ can be chosen between $b_{n}=(-1)^{n}$ or $b_{n}=(-1)^{n+1}$, so that it can be set as $b_{0}=1$ or $b_{0}=-1$ for a given initial condition $x_{0}$. The parameter $\epsilon$ controls the amplitude of the perturbation and $R$ is a control parameter of the system. For $R>0$ the mapping is dissipative and the system presents attractors. $x_{n}$ is a dynamical variable evaluated at the $n$th iteration of the mapping. For $\epsilon=0$ the traditional second degree logistic mapping is recovered. Since from May [28], this mapping has been used to move forward an avalanche of extremely important results in dynamical systems and theory of nonlinear systems.

In the time perturbed logistic map, orbits of period $k$ are obtained for $x_{n+k}=x_{n}$ and $b_{n+k}=b_{n}$. For odd $k,\left|b_{n+k}-b_{n}\right|=2$ whereas for even $k$ we have $b_{n+k}-b_{n}=0$. Thereby, Eq. (9) presents only periodic orbits with even period. Thus, solutions of Eq. (3) are written as

$x_{ \pm}^{*}=\frac{1}{2}$,

where, here, the subscript indicates the signal of $b$ and $x_{-}^{*}=$ $F^{\left(k_{1}\right)}\left(x_{+}^{*}\right)$ and $x_{+}^{*}=F^{\left(k_{2}\right)}\left(x_{-}^{*}\right)$ are mappings of $k$-extreme orbits. Then, a $k$-extreme curve is a $k$-superstable curve for even values of $k$.

The 1-extreme curve is obtained substituting $x_{ \pm}^{*}$ in $x_{n}$ and $x_{n+1}$ of Eq. (9), yielding in

$\epsilon=\frac{1}{b}\left(\frac{2}{R}-1\right)$.

These curves are shown in Fig. 2(b) labeled as (1)_ (blue curve) and $(1)_{+}$(cyan curve) for $x_{-}^{*}$ and $x_{+}^{*}$, respectively. The number inside of the parenthesis identifies the 1-extreme orbit.

The 2-extreme curve is a 2-superstable curve, since $k$ is even, and can be obtained considering the second iteration of system (9),

$x_{n+2}=u v x_{n}\left[\left(1-x_{n}\right)-x_{n} u\left(1-x_{n}\right)^{2}\right]$,

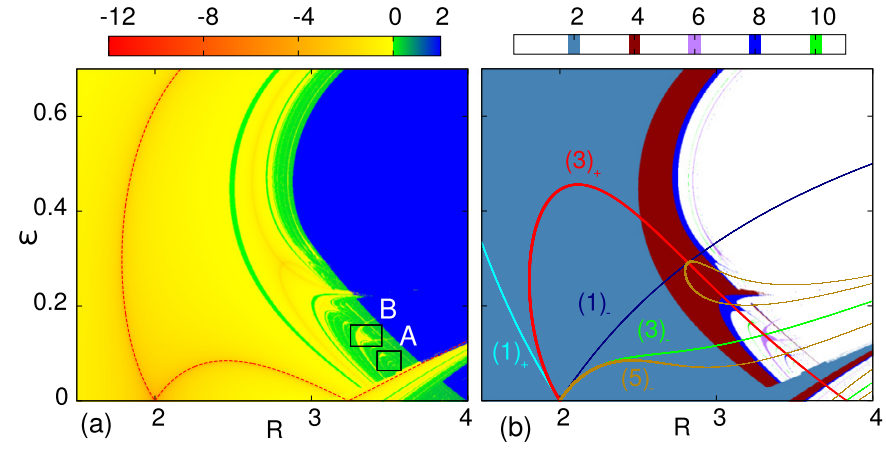

Fig. 2. (Color online.) Plot of the parameter space $\epsilon$ vs. $R$ where the colors define: (a) Lyapunov exponents; (b) Periods. The lines in (b) represent the $k$-extreme orbits Initial conditions with $x_{0}=0.4$ were used.

with $u=R(1+b \epsilon), v=R(1-b \epsilon)$. Thus,

$R=\frac{u+v}{2}$ and $\epsilon=\frac{u-v}{b(u+v)}$.

Applying the superstability condition $x_{n+2}=x_{n}=x_{ \pm}^{*}$ to Eq. (12), we get $v=\frac{2}{u\left(1-\frac{u}{4}\right)}$. The 2-superstable curves are obtained varying $u$ to determine $v$ and taking these values to Eq. (13). Fig. 2(a) shows the 2-superstable curves (dashed red lines).

The 3-extreme curves are obtained substituting $x_{ \pm}^{*}$ in $x_{n}$ and $x_{n+3}$ in the map resulted after the third iteration of system (9). The parameters $R$ and $\epsilon$ are determined by Eqs. (13) where now,

$u=\frac{1}{2 q(1-q)} \quad$ and $\quad v=\frac{4 q}{u\left(1-\frac{u}{4}\right)}$.

The 3-extreme curves are obtained changing $q$, to calculate $u$ and $v$, and substituting in Eqs. (13). These curves are shown in Fig. 2(b) colored in red $\left[(3)_{+}\right]$and in green [(3)- ]. The other extreme curves $(k)_{ \pm}$are obtained numerically.

Computing the analytical results we can verify, by a direct analysis of Eq. (11), that the 1-extreme curves $(1)_{ \pm}$intercept each other at $(R, \epsilon)=(2,0)$. This is the same point where the two 2-superstable curves intersect, as can be verified both analytically, for $u=2$ (which implies $v=2$ ) in Eqs. (13), and numerically, as shown in Fig. 2(b). Finally, considering $q=\frac{1}{2}$ in Eqs. (14) to calculate $u$ and $v$ and, substituting in Eqs. (13), we obtain that the curves $(3)_{+}$and $(3)_{-}$are also crossing the point $(R, \epsilon)=(2,0)$. Consequently, a superstable orbit of period 6 is formed. Since a period 2 orbit is also periodic of 6 iterations, the results are in total agreement with the discussion in section 1. For the same reason, all extreme orbits cross this point [see Fig. 2(b) for some examples].

When two extreme orbits $\left(k_{1}\right)_{ \pm}$and $\left(k_{2}\right)_{\mp}$ intercept each other, a period $k=\left(k_{1}+k_{2}\right)$-superstable orbit is observed. In Fig. 2(b), periodic regions are highlighted in colors and the curves (1) _ and $(3)_{+}$intersect each other in a region of periodicity $4(1+3$ of the two 1- and 3-extreme curves), exactly in the same point where the superstable curves are crossing each other in Fig. 2(a). The same is true for the intersections between curves (3) _ and (5) _ with the curve $(3)_{+}$. An enlargement of region A shown in Fig. 2(a) produces Figs. 3(a) and (b). Here, one sees that the curve (3)+ gives the organization of the periodic regions in this cascade. The interception of the curves (3) + and (5) - happens exactly in the periodic region with period 8 (blue region). The extreme curves (7) and $(3)_{+}$produce a periodic region with period 10 [green region in Fig. 3(b)]. The important result, confirmed in our simulations, is that the interception of two extreme curves with different initial conditions always produces a periodic region. Such observation can be continually confirmed noticing the interception between curve 


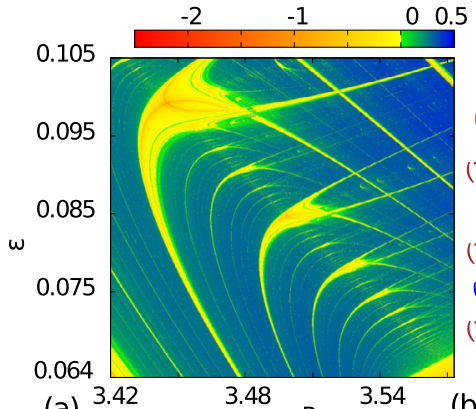

(a) 3.42

$\mathrm{R}$

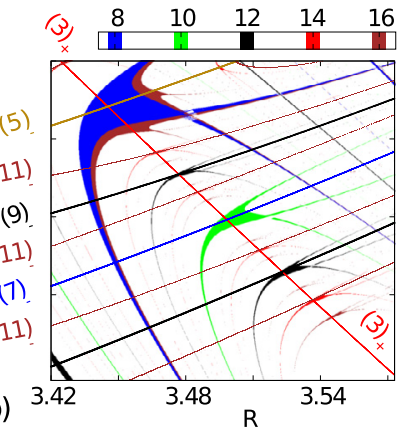

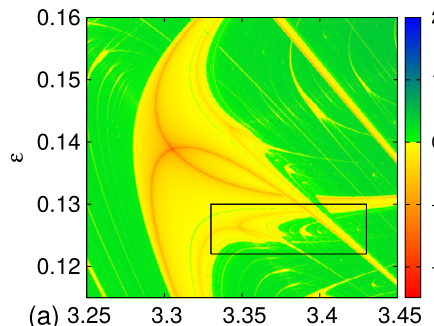
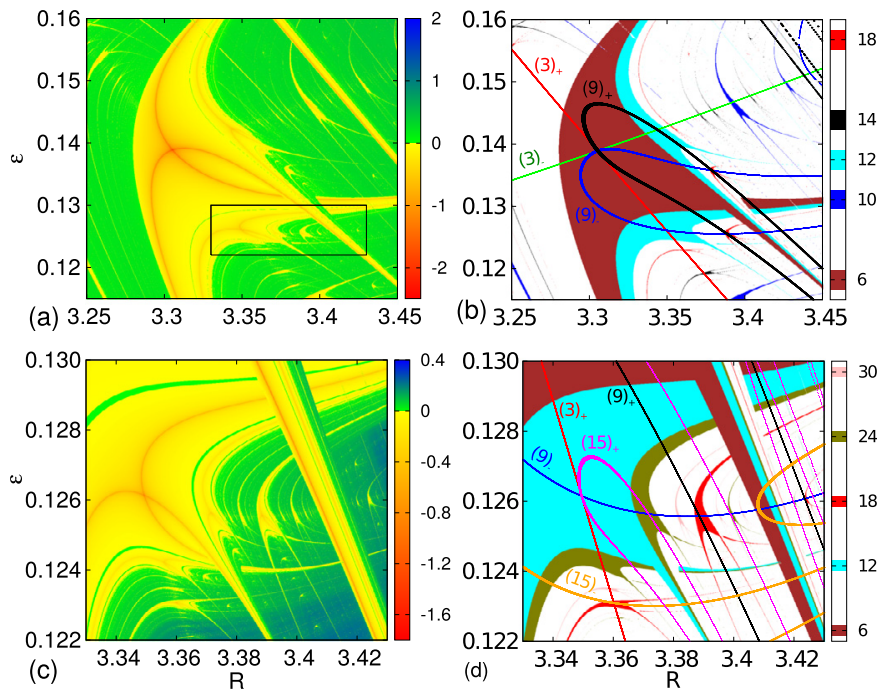

(3) + with curves (9) $)_{-}$and $(11)_{-}$, which show the position of periodic regions with periods 12 and 14. Many other extreme orbits exist in the parameter space, however we decided to show only these curves. The remarkable conclusion is that extreme curves are not limited to organize cascades, but they introduce the topological characteristic of the superstable orbits in the whole cascade. In this case, all superstable orbits have incorporated in their trajectories the 3-extreme orbit.

The curves (3) + and (3) $)_{-}$in Fig. 2(b) intercept each other in the periodic region with period 6. An enlargement of region B from Fig. 2(a) shows it in details, as can be seen from Figs. 4(a) and (b). At this figure, one sees that (3) + and (3) _ align different cascades of periodic regions. (9) + and $(9)_{-}$, as expected, intercept each other at the same point of (3) + and (3) $)_{-}$, and also align some cascades. These cascades of complicate structures of periodicity are largely observed without connection between them [16,17,20,29]. Here, the 9-extreme orbits associate clearly these cascades with the shrimp characterized by period 6 [brown region in Fig. 4(b)] in the same way as the cascades organized by (3) + are associated with the complicate set of periodicity 2 shown in Fig. 2(b).

In Figs. 4(c) and (d), which are an enlargement of the rectangle shown in Fig. 4(a), it is shown with details the cascades that curves $(3)_{+}$and (9) - create. An important result here is to observe in Fig. 4(d) that the interception of curves (9)_ (blue line) and (15)_ (orange line) does not generate the center of a periodic region, it is intercepting only one superstable curve with periodicity $6(15-9)$ (brown region). To explain this, consider that $x_{+}^{*}=F^{\left(k_{1}\right)}\left(x_{-}^{*}\right)$ and $x_{+}^{*}=F^{\left(k_{2}\right)}\left(x_{-}^{*}\right)$, with $k_{2}>k_{1}$. Then, $F^{\left(k_{1}\right)}\left(x_{-}^{*}\right)=$ $F^{(k)}\left(x_{-}^{*}\right)=F^{\left(k_{2}-k_{1}\right)+k_{1}}\left(x_{-}^{*}\right)=F^{\left(k_{2}\right)}\left(F^{\left(k_{2}-k_{1}\right)}\left(x_{-}^{*}\right)\right)$ and we conclude that $x_{-}^{*}=F^{\left(k_{2}-k_{1}\right)}\left(x_{-}^{*}\right)$ which is a superstable orbit period $k_{2}-k_{1}$.

\section{Summary and conclusions}

Sets of periodicity that are continuously connected (where the periodic orbit changes smoothly) can be identified by the crossing of superstable curves as shrimps connected along spirals [10]. This is different for discrete (non-connected) sets of periodicity. For this case, we have shown that extreme orbits play a major role in the organization of periodic structures in the parameter space of onedimensional dissipative mappings. Firstly, they localize accurately a complex structure of periodicity, even for high period orbits. They also highlight where complicate periodic regions exist giving the directions of cascades of periodic regions and exposing their global distribution in the parameter space. The extreme curves give the possibility to understand deeper the organization of periodicity. Here we connect two cascades organized by 9-extreme orbits with a shrimp via a crossing the 3-extreme orbits. Therefore, the presented discussion is an important tool to investigate both global and local organizations of complex structures of peri-

Fig. 4. (Color online.) Plot of the parameter space $\epsilon$ vs. $R$ where the colors define the Lyapunov exponents in (a) and (c); Periods in (b) and (d). The lines in (b) and (d) represent the $k$-extreme orbits. The parameter spaces in (a) and (b) correspond to an enlargement of region B of Fig. 2(a). Plots (c) and (d) correspond to a enlargement of the rectangle shown in (a). Initial condition was $x_{0}=0.4$

odicity in parameter space. As applications, the circle map and also a time perturbed logistic map were studied. It is worth to comment that there are systems where complicate sets of periodicity do not show intersections between superstable curves, commonly in higher dimension [5], which is an obstacle in applying the concept of extreme orbits. However, the scenario around these sets has several similarities with the ones described here.

\section{Acknowledgements}

DRC acknowledges Brazilian agency FAPESP (2013/22764-2). $\mathrm{MH}$ thanks CAPES. GG thanks CNPq. ROMT thanks CNPq (205875/2014-9), FAPESP (2014/22445-7) and EU Marie-Curie IRSES Brazilian-European partnership in Dynamical Systems (FP7-PEOPLE-2012-IRSES 318999 BREUDS). EDL thanks CNPq, FUNDUNESP and FAPESP (2012/23688-5), Brazilian agencies. This research was supported by resources supplied by the Center for Scientific Computing (NCC/GridUNESP) of the São Paulo State University (UNESP).

\section{References}

[1] V. Arnold, Izv. Akad. Nauk SSSR, Ser. Mat. 25 (1961) 21.

[2] J. Gallas, Phys. Rev. Lett. 70 (1993) 2714

[3] E. Barreto, B.R. Hunt, C. Grebogi, J.A. Yorke, Phys. Rev. Lett. 78 (1997) 4561.

[4] D.M. Maranhão, M.S. Baptista, J.C. Sartorelli, I.L. Caldas, Phys. Rev. E 77 (2008) 037202

[5] W. Façanha, B. Oldeman, L. Glass, Phys. Lett. A 377 (2013) 1264.

[6] C. Manchein, A. Celestino, M.W. Beims, Phys. Rev. Lett. 110 (2013) 114102.

[7] P. Gaspard, R. Kapral, G. Nicolis, J. Stat. Phys. 35 (1984) 697.

[8] O. de Feo, G.M. Maggio, Int. J. Bifurc. Chaos 13 (2003) 2917.

[9] C. Bonatto, J.A.C. Gallas, Phys. Rev. Lett. 101 (2008) 054101.

[10] R. Barrio, F. Blesa, S. Serrano, Phys. Rev. Lett. 108 (2012) 214102.

[11] R. Stoop, P. Benner, Y. Uwate, Phys. Rev. Lett. 105 (2010) 074102.

[12] Y. Kuznetsov, Elements of Applied Bifurcation Theory, vol. 112, Springer-Verlag, New York, 2004.

[13] R.O. Medrano-T, M.S. Baptista, I.L. Caldas, Chaos 15 (2005) 033112.

[14] R. Barrio, F. Blesa, S. Serrano, A. Shilnikov, Phys. Rev. E 84 (2011) 035201(R).

[15] R. Vitolo, P. Glendinning, J.A.C. Gallas, Phys. Rev. E 84 (2011) 016216.

[16] C. Bonatto, J. Gallas, Philos. Trans. R. Soc. Lond. A 366 (2008) 505.

[17] S.L.T. de Souza, A.A. Lima, I.L. Caldas, R.O. Medrano-T, Z.O. Guimarães-Filho, Phys. Lett. A 376 (2012) 1290.

[18] L. Glass, Nature 410 (2001) 277.

[19] K. Kaneko, Prog. Theor. Phys. 68 (1982) 669. 
[20] V. English, U. Parlitz, W. Lauterborn, Phys. Rev. E 92 (2015) 022907.

[21] E.S. Medeiros, R.O. Medrano-T, I.L. Caldas, S.L.T. de Souza, Phys. Lett. A 377 (2013) 628.

[22] E.N. Lorenz, Physica D 237 (2008) 1689.

[23] H. Bai-Lin, Elementary Symbolic Dynamics and Chaos in Dissipative Systems, World Scientific Publishing Co. Inc., Singapore, 1989.

[24] S. Fraser, R. Kapral, Phys. Rev. A 25 (1982) 3223.
[25] W.Z. Zeng, L. Glass, Physica D 40 (1989) 218

[26] Edson D Leonel, J. Kamphorst Leal da Silva, S. Oliffson Kamphorst, Physica A 295 (2001) 280-284.

[27] Edson D. Leonel, J. Kamphorst Leal da Silva, S. Oliffson Kamphorst, Int. J. Bifurc. Chaos 12 (7) (2002) 1667.

[28] R. May, Nature 261 (1976) 459.

[29] D.F.M. Oliveira, E.D. Leonel, New J. Phys. 13 (2011) 123012. 